\title{
Midline Supraumbilical Incision as an Option for Morbidly Obese Patients? Case Report
}

\author{
Janete Vettorazzi1,2,3*, Gabriela Françoes Rostirolla1,4, Gabrielle Soares Behenck ${ }^{3}$, \\ Fernanda Oliveira Castilhos ${ }^{1,4}$, Eduardo Vettorazzi-Stuczynski4, Edimárlei Gonsales Valério ${ }^{1,2,3}$ \\ ${ }^{1}$ Postgraduate Program in Health Sciences: Gynecology and Obstetrics, Universidade Federal do Rio Grande do Sul (UFRGS), \\ Porto Alegre, Brasil \\ ${ }^{2}$ Department of Gynecology and Obstetrics, Faculdade de Medicina (FAMED), Universidade Federal do Rio Grande do Sul, Porto \\ Alegre-Rio Grande do Sul (RS), Brasil \\ ${ }^{3}$ Service of Obstetrics and Gynecology, Hospital de Clínicas de Porto Alegre, Porto Alegre/RS, Brasil \\ ${ }^{4}$ Hospital Geral de Caxias do Sul e Faculdade de Medicina da Universidade de Caxias do SUl, Caxias do Sul/RS, Brasil \\ Email: *jvettorazzi@hcpa.edu.br
}

How to cite this paper: Vettorazzi, J., Rostirolla, G.F., Behenck, G.S., Castilhos, F.O., Vettorazzi-Stuczynski, E. and Valério, E.G. (2021) Midline Supraumbilical Incision as an Option for Morbidly Obese Patients? Case Report. Open Journal of Obstetrics and $G y$ necology, 11, 1517-1523.

https://doi.org/10.4236/ojog.2021.1111143

Received: October 4, 2021

Accepted: November 15, 2021

Published: November 18, 2021

Copyright (c) 2021 by author(s) and Scientific Research Publishing Inc. This work is licensed under the Creative Commons Attribution International License (CC BY 4.0).

http://creativecommons.org/licenses/by/4.0/

\begin{abstract}
Background: Nowadays we observe growing rates of obesity, mainly among women. It has a great impact on maternal and fetal morbimortality and requires a specific obstetric approach. Aim: To discuss different approaches to abdominal incision in cesarean section in obese patients. Case presentation: Patient with morbid obesity submitted to an elective cesarean section due to fetal macrosomia and who underwent a cesarean section with a supraumbilical median incision evolving without any postpartum complications. Conclusion: It's important to evaluate and chose the best incision in morbidly obese pregnant women aiming to reduce difficulties in fetal extraction and postoperative complications. Midline supraumbilical incision is a good choice in these cases, reducing surgical time and postpartum hemorrhage.
\end{abstract}

\section{Keywords}

Obesity, Caesarean Section, Surgical Incision, Abdominal Obesity

\section{Introduction}

Recent data have shown the rise in global obesity at a pandemic level. In Brazil, data from 2015 demonstrated a prevalence of approximately $60 \%$ of the total population in the overweight and obesity range [1]. Weight gain has been observed in all age groups, being more prevalent among women. Many of these women are in the reproductive age, which leads to an increase in the number of high-risk pregnancies and, consequently, maternal and fetal morbidity and 
mortality.

It is known that obese women are at increased risk of complications related to pregnancy, such as anemia, hypertension, preeclampsia, premature birth, emergency cesarean section and gestational diabetes mellitus, among others [2]. All pregnant women with obesity should be followed up in appropriate prenatal services in order to manage the complications that may arise during pregnancy and to plan delivery correctly.

In cases where cesarean section is needed in obese patients, planning and adaptation of obstetric management from prenatal care to birth are required, including a special surgical table, adequate anesthetic evaluation and differentiated surgical material, as well as changes in the surgical technique with choice of surgical incision for cesarean section.

\section{Objective}

To report the case of a patient with morbid obesity (BMI over $40 \mathrm{~kg} / \mathrm{m}^{2}$ ) and other associated comorbidities who was hospitalized for elective interruption of pregnancy due to fetal macrosomia and underwent a cesarean section with a supraumbilical midline incision. The objective is to bring to discussion the possibility of new abdominal incisions in cesarean section in obese patients, aiming the reduction of pre and postoperative complications, and, consequently, a decrease in maternal and fetal morbidity and mortality in these patients.

\section{Case Report}

A 31-year-old gravida 2 para 1, patient at the high-risk pregnancy prenatal care in our hospital due to morbid obesity (grade III), hypothyroidism, type 2 diabetes mellitus and chronic hypertension. She was under the use of several medications (levothyroxine $75 \mathrm{mcg}$ daily, methyldopa $1 \mathrm{~g}$ twice a day, metformin $850 \mathrm{mg}$ twice a day, calcium carbonate $500 \mathrm{mg}$ twice a day and aspirin $100 \mathrm{mg}$ once a day). It was opted to start insulin at 30 weeks of gestational age due to a poor control in diabetes, with altered glycemic levels and fetal macrosomia. At 37 weeks of gestational age she was sent to the obstetric center due to high blood pressure at prenatal care appointment. Her tests came positive for pre-eclampsia and she was admitted. During hospitalization, she went through serial blood tests and monitoring of blood pressure, glucose levels and fetal well-being assessment. She didn't present any other complications before delivery. At 38 weeks of gestational age it was decided for elective interruption due to pre-eclampsia. The obstetric ultrasound performed during hospitalization showed a cephalic fetus, with estimated fetal weight at 5550 grams. Therefore, due to fetal macrosomia, it was chosen to perform a cesarean section.

The patient had a body mass index of $49 \mathrm{~kg} / \mathrm{m}^{2}$ and an adipose layer estimated around $30 \mathrm{~cm}$ thick. Different surgical approaches were considered and discussed with the patient and surgical staff, but because of the fact that the patient presented an apron-like panniculus in the abdomen, it was decided to perform a 
supraumbilical incision. She went through a laparotomy with midline supraumbilical incision approximately $15 \mathrm{~cm}$ long (Figure 1), with good exposure of the uterus (Figure 2). It was performed a segmental hysterotomy, without difficulties in fetal extraction. The baby was born with 5595 grams and 2 and 7 apgar score in the 1st and 5th minute. She presented with intraoperative blood loss due to uterine atony, controlled with uterine massage, $20 \mathrm{IU}$ of oxytocin in saline solution, $1 \mathrm{~g}$ of tranexamic acid and $800 \mathrm{mcg}$ of misoprostol and that didn't require other surgical approaches. The closure was made according to our hospital's protocol: the uterus was closed with a double layer continuous suture with polyglactin 910 size 0 ; the aponeurosis was closed with polyglactin 910 size 0 ; for the subcutaneous layer it was used polyglactin 910 size 2.0, and for the skin, nylon non absorbable suture size 4.0. The procedure occurred without other complications.

She had a good postpartum evolution, without postoperative pain or signs of infection (Figure 3). The uterine atony didn't have severe repercussions in hemoglobin levels. She was discharged together with her baby within 72 hours without surgical complications during hospital stay. She returned after 10 days to remove surgical stitches. She didn't present any signs of wound infection, dehiscence, or any other complications. A verbal and sign consent was obtained with the patient to publish this case report.

\section{Discussion}

In pregnant patients with morbid obesity, the choice of skin incision is important to reduce difficulty in fetal extraction and post operative complications, including seroma, wound infection, blood transfusion, among others. However, the type of incision that has better results is still controversial.

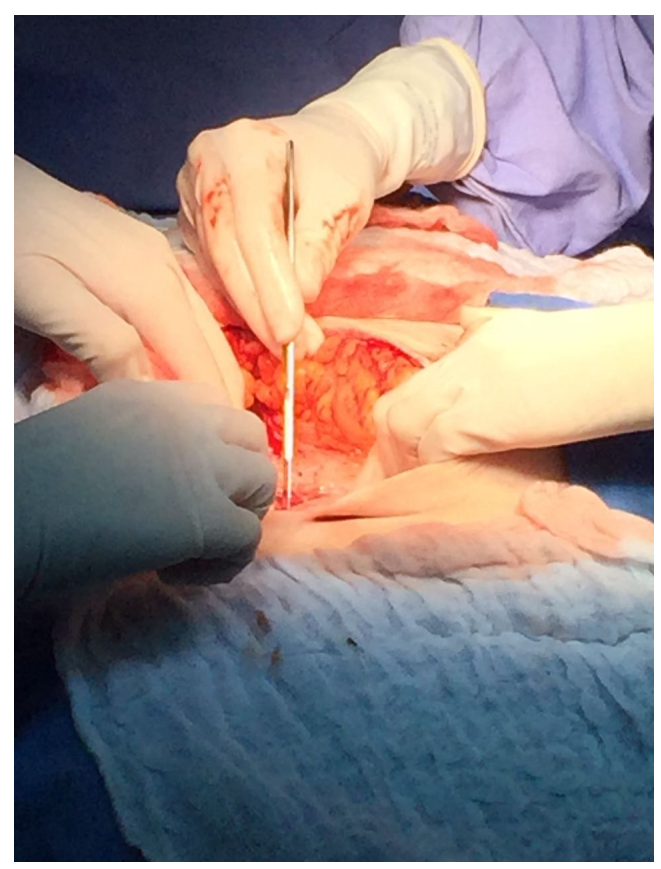

Figure 1. Midline supraumbilical incision. 


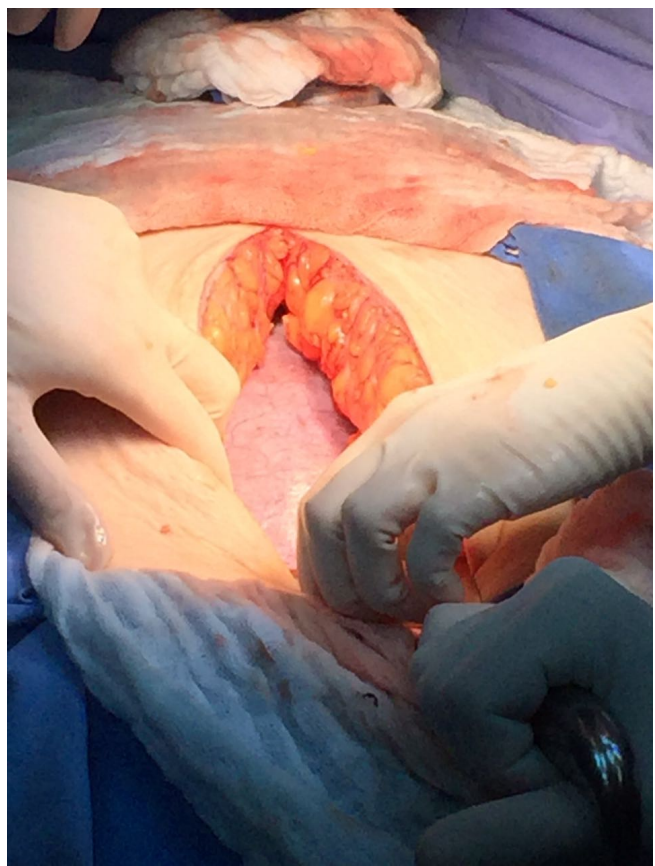

Figure 2. Uterus.

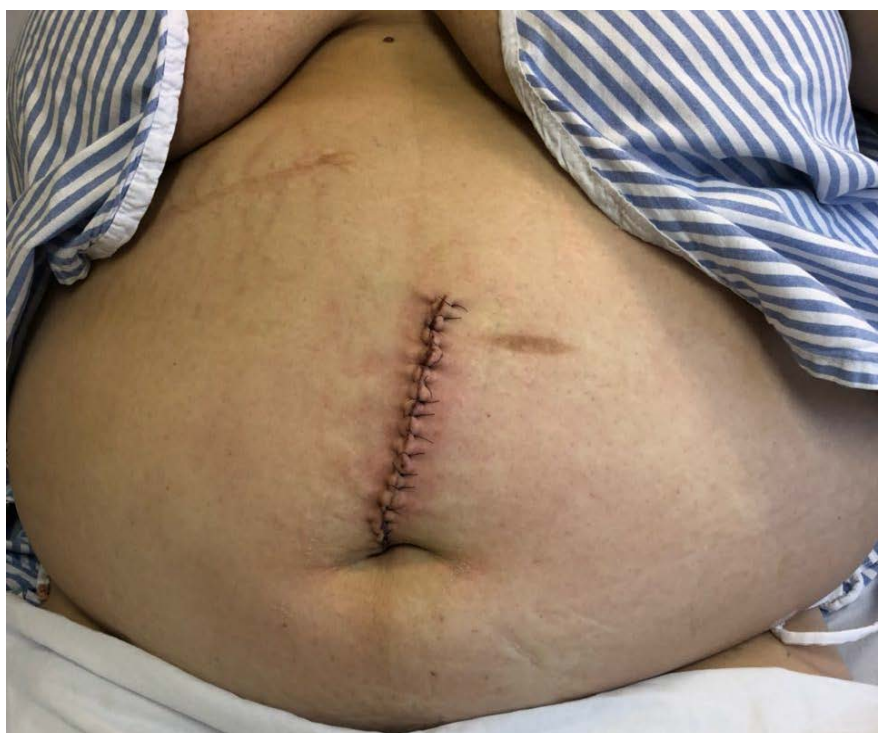

Figure 3. Third day post-operatory.

Transverse incisions are usually preferred for presenting better cosmetic appearance, less postoperative pain and less hernia formation compared with vertical incisions [3]. The Pfannenstiel incision is made 2 to $3 \mathrm{~cm}$ above the symphysis pubis and has a curved shape. The Joel-Cohen incision is straight, made 3 $\mathrm{cm}$ below the line that joins the anterior superior iliac spines, positioned over the Pfannenstiel incision. In some studies the Joel-Cohen incision has shown some advantages compared to the Pfannenstiel incision, such as lower rates of fever, postoperative pain requiring medication and blood loss. However, the Pfannenstiel incision is usually preferred because of the better cosmetic result 
[4]. Additionally, the type of skin incision made at cesarean section has influence on the type of uterine incision, and it was observed an increase in vertical uterine incisions in patients who underwent vertical skin incisions, compared with low transverse skin incisions [5], causing an impact in future pregnancies. However, a possible explanation for this correlation is that in some studies, such as the one conducted by Houston et al. [6], all obese women went through a cesarean section with supraumbilical midline incision, even the patients that didn't have an abdominal panniculus. In these cases, the uterus had to be approached from the fundus.

There are few studies comparing the complication rates between the different tipes of incision in cesarian section in obese women. The majority of studies compare the Pfannenstiel incision with infraumbilical midline incision, with few studies evaluating the results of midline supraumbilical incision. Also, usually the patients that are chosen to have a supraumbilical midline incision are heavier than those who deliver through a transverse incision, making it hard to compare the results and complications of this type of incision without bias.

In some patients with morbid obesity, we observe a higher risk in infection due to the humidity caused by the panniculus covering the incision and promoting the growth of anaerobic bacteria. A pilot study showed that patients with class III obesity that had high transverse skin incisions, instead of low transverse incisions, had a decreased risk of wound complications and endometritis [7]. In contrast, a recent systematic review showed that all types of vertical incisions had association with increased risk of wound complications, compared with all types of transverse incisions. However, this finding could be due to the fact that the patients that received vertical incisions had more risk factors, such as increased BMI and diabetes, compared to the other group [8].

Additionally, the thick abdominal wall can impair the fetal extraction, and in patients with an apron-like panniculus in abdomen, it can be hard to identify anatomic landmarks to locate intra abdominal organs, making it difficult to decide the exact place to incise. In these cases, it's important to evaluate the possibility of other surgical approaches that offer lower risks to the patient and the fetus. In a series of cases that showed good results with this kind of incision, they examined every patient in standing position and in dorsal decubitus, in order to determine the site of the incision depending on the projection of the pubic symphysis [9]. This is an interesting approach to help define which kind of skin incision is best for each patient.

We highlight the fact that this is not the usual approach to obese patients going under cesarean section in our institution, even the ones with class III obesety or an "apron-like" abdomen. In the last five years, this was the only case using this approach. However, In this case report, we considered the midline supraumbilical incision an interesting choice, since the patient presented a thick abdominal wall with an apron-like panniculus covering the pubic symphysis, making it hard to identify surgical landmarks. Additionally, the cesarean section was indicated in this case due to fetal macrosomia, requiring planning the sur- 
gical approach expecting a difficult fetal extraction. In this case, the incision made provided good visualization of the uterus, which favored the fetal extraction. Also, the patient didn't present any signs of wound infection, which was a major worry in this case because of her comorbidities.

Midline supraumbilical incision in obese patients with "apron-like" panniculus apears to be a good option to access the abdominal cavity and to extract de fetus. This type of incision usually provides a better exposure of the surgical field, because the tissues cut where the fat layer is least thick, also reducing the risk of parietal complications [2]. More studies are still required to compare the different types of incision and their benefits and complications in each case.

\section{Conclusion}

The midline supraumbilical skin incision in pregnant patients with morbid obesity is an option that should be considered and discussed with the patient. This incision can reduce operating time and improve the exposure of the surgical field, facilitating access to the abdominal cavity and fetal extraction, also reducing bleeding. More studies are required comparing maternal and fetal outcomes in different incisions in obese patients.

\section{Conflicts of Interest}

The authors declare no conflicts of interest regarding the publication of this paper.

\section{References}

[1] Pesquisa do IBGE mostra aumento da obesidade entre adultos. Gov.br, Brasil, 21 de out de 2020.

https://www.gov.br/pt-br/noticias/saude-e-vigilancia-sanitaria/2020/10/pesquisa-do -ibge-mostra-aumento-da-obesidade-entre-adultos

[2] Mathai, M., Hofmeyr, G.J. and Mathai, N.E. (2013) Abdominal Surgical Incisions for Caesarean Section. Cochrane Database of Systematic Reviews, 5, CD004453. https://doi.org/10.1002/14651858.CD004453.pub3

[3] Bickenbach, K.A., Karanicolas, P.J., Ammori, J.B., Jayaraman, S., Winter, J.M., Fields, R.C., Govindarajan, A., Nir, I., Rocha, F.G. and Brennan, M.F. (2013) Up and down or Side to Side? A Systematic Review and Meta-Analysis Examining the Impact of Incision on Outcomes after Abdominal Surgery. The American Journal of Surgery, 206, 400-409. https://doi.org/10.1016/j.amjsurg.2012.11.008

[4] Gizzo, S., Andrisani, A., Noventa, M., Di Gangi, S., Quaranta, M., Cosmi, E., D’Antona, D., Nardelli, G.B. and Ambrosini, G. (2015) Caesarean Section: Could Different Transverse Abdominal Incision Techniques Influence Postpartum Pain and Subsequent Quality of Life? A Systematic Review. PLOS ONE, 10, e0114190. https://doi.org/10.1371/journal.pone.0114190

[5] Bell, J., Bell, S., Vahratian, A. and Awonuga, A.O. (2011) Abdominal Surgical Incisions and Perioperative Morbidity among Morbidly Obese Women Undergoing Cesarean Delivery. European Journal of Obstetrics \& Gynecology and Reproductive Biology, 154, 16-19. https://doi.org/10.1016/j.ejogrb.2010.07.043

[6] Houston, M.C. and Raynor, B.D. (2000) Postoperative Morbidity in the Morbidly 
Obese Parturient Woman: Supraumbilical and Low Transverse Abdominal Approaches. American Journal of Obstetrics \& Gynecology, 182, 1033-1035. https://doi.org/10.1067/mob.2000.105400

[7] Walton, R.B., Shnaekel, K.L., Ounpraseuth, S.T., Napolitano, P.G. and Magann, E.F. (2019) High Transverse Skin Incisions May Reduce Wound Complications in Obese Women Having Cesarean Sections: A Pilot Study. The Journal of Maternal-Fetal \& Neonatal Medicine, 32, 781-785. https://doi.org/10.1080/14767058.2017.1391780

[8] Mccurdy, R.J., Felder, L.A., Saccone, G., Edwards, R.K., Thornburg, L.L., Marrs, C., Conner, S.N., Strauss, R. and Berghella, V. (2020) The Association of Skin Incision Placement during Cesarean Delivery with Wound Complications in Obese Women: A Systematic Review and Meta-Analysis. The Journal of Maternal-Fetal \& Neonatal Medicine, 1-13. https://doi.org/10.1080/14767058.2020.1786050

[9] Tixier, H., Thouvenot, S., Coulange, L., Peyronel, C., Filipuzzi, L., Sagot, P. and Douvier, S. (2009) Cesarean Section in Morbidly Obese Women: Supra or Subumbilical Transverse Incision? Acta Obstetricia et Gynecologica Scandinavica, 88, 1049-1052. https://doi.org/10.1080/00016340903128462 